\title{
Plasma chemistry for development of novel materials
}

\author{
MEGURU TEZUKA \\ Saitama Institute of Technology, 1690 Fusaiji, Okabe, Saitama 369-02 Japan
}

\begin{abstract}
The possibilities of plasma chemistry as a novel synthetic method in the reaction fields of material science are discussed, stressing the fundamental aspect. The characteristics of the chemical process using nonequilibrium plasmas are described and some typical examples of the application of plasma techniques to organic chemistry are reviewed. The current approaches to improve the selectivity in plasma synthesis are also mentioned.
\end{abstract}

Keywords. Plasma chemistry; nonequilibrium discharge; organic plasma reactions; plasma-liquid interaction.

\section{Introduction}

As a field of research plasma chemistry has had a long history and has demonstrated the potential of plasmas for promoting a wide variety of reactions, but the development of practical applications has been relatively slow. The last several decades, however, have seen the remarkable progress of a number of such applications in the fields of organic chemistry, polymer chemistry, solid-state electronics, metallurgy, and environmental chemistry. In table 1 are listed the categories of sessions opened at the 12th International Symposium on Plasma Chemistry, which was held in August 1995, and the number of papers contributed to each session. We can see that plasma chemistry covers some of the most important and intriguing research fields in material science. Recently, application of plasma techniques to environmental issues is also attracting growing interest among researchers. Although each of these advances is worth noting, from the viewpoint of exploiting novel methods for chemical synthesis, special attention should be devoted to those in which the plasma is used because of its unique physical characteristics rather than as a source of heat. For this reason, in this paper, the author would like to describe chemical processes using nonequilibrium plasmas, focusing on the fundamental aspect. Applications of high-temperature equilibrium plasmas will be excluded since the principal use of such plasmas is as a source of high-temperature heat.

\section{Chemical processes in plasmas}

The field of plasma chemistry deals with the occurrence of chemical reactions in a partially ionized gas composed of ions, electrons, and neutral species. This state of matter can be produced through the action of either very high temperatures or strong electric or magnetic fields. In a gaseous electric discharge, free electrons gain energy from an imposed electric field and lose this energy through collisions with neutral gas molecules. The transfer of energy to the molecules leads to the formation of a variety of new species including metastables, atoms, free radicals, and ions. These products are all active 
Table 1. 12th International Symposium on Plasma Chemistry.

\begin{tabular}{llc}
\hline & Session & No. of papers \\
\hline 1. & Large area plasma source & 10 \\
& Diagnostics and modelling & 21 \\
& Characterization of basic processes in plasmas & 88 \\
2. $\quad$ Bulk plasma synthesis & 14 \\
& Plasma metallurgy & 11 \\
& Plasma heat transfer & 33 \\
& Synthesis of particles and clusters & 61 \\
Plasma spraying & 19 \\
3. Etching & 14 \\
& Plasma-surface interactions & 29 \\
& Plasma polymerization & 39 \\
& Surface modification & 22 \\
4. Plasma CVD & Environmental applications & 82 \\
\hline
\end{tabular}

August 21-25, 1995 at Minneapolis, USA.

chemically and thus can serve as precursors to the formation of new stable compounds.

$$
\begin{aligned}
\mathbf{M}+e^{*} & \rightarrow \mathbf{M}^{+} \\
& \rightarrow \mathbf{M}^{-} \\
& \rightarrow \mathbf{M}^{*}
\end{aligned}
$$

A number of man-made as well as naturally occurring plasmas can be characterized in terms of the electron temperature and density. The two regions of greatest interest to plasma chemistry are those labeled "glow discharges" and "arcs". In table 2, the physical characteristics of both plasmas along with those of the "corona" are summarized. In a glow discharge, average electron energies are $1-10 \mathrm{eV}$, and electron densities, $10^{9}-10^{12} \mathrm{~cm}^{-3}$. An additional characteristic of such plasmas is the lack of equilibrium between the electron temperature $T_{e}$ and the gas temperature $T_{g}$. The absence of thermal equilibrium makes it possible to obtain a plasma in which the gas temperature may be near ambient values at the same time that the electrons are sufficiently energetic to cause a rupture of molecular bonds. This feature makes glow-discharge plasmas well suited for the promotion of chemical reactions involving thermally sensitive materials. By contrast, in the plasmas produced by arcs or plasma jets, an equilibrium situation is established in which the electron and gas temperatures are nearly identical. The very high gas temperature $\left(>5 \times 10^{3} \mathrm{~K}\right)$ in such plasmas makes them suitable for processing inorganic materials and organic compounds with extremely simple structures. More complex organic materials and polymers cannot be treated under these conditions since they would be rapidly degraded.

\section{Applications to organic chemistry}

For the reasons mentioned above, nonequilibrium plasmas have been usually employed for applications in organic chemistry. Here, the molecules might be activated through collisions with energetic electrons rather than by thermal action. In practice, a number of interesting organic transformations which can hardly be accomplished by conventional chemical methods have taken place in such plasmas. The characteristics of plasma 
Table 2. Physical characteristics of plasma.

\begin{tabular}{llccccc}
\hline Plasma & Discharge & $\begin{array}{c}\langle n\rangle^{\mathrm{a}} \\
\left(\mathrm{cm}^{-3}\right)\end{array}$ & $\begin{array}{c}E^{\mathrm{b}} \\
\left(\mathrm{Vcm}^{-1}\right)\end{array}$ & $\begin{array}{c}p^{\mathrm{c}} \\
(\mathrm{mmHg})\end{array}$ & $\begin{array}{c}T_{e}^{\mathrm{d}} \\
(\mathbf{K})\end{array}$ & $\begin{array}{c}T_{\mathrm{g}}^{\mathrm{e}} \\
(\mathbf{K})\end{array}$ \\
\hline Thermal & Arc & $10^{14}<$ & $<20$ & $100<$ & $\sim 10^{4}$ & $\sim 10^{4}$ \\
Low temperature & Glow & $10^{9}-10^{12}$ & $50-100$ & $<100$ & $\sim 10^{4}$ & $\sim 7 \times 10^{2}$ \\
& Corona & $<10^{16}$ & $2 \times 10^{4}<$ & $100<$ & $\sim 10^{4}$ & $\sim 4 \times 10^{2}$ \\
\hline
\end{tabular}

${ }^{a}$ Electron density; ${ }^{b}$ strength of electric field; ${ }^{c}$ pressure; ${ }^{d}$ electron temperature; ${ }^{e}$ gas temperature

techniques as a preparative tool in organic chemistry might be summarised as follows: (1) the chemical process in a nonequilibrium plasma normally comprises the homogeneous gas-phase reactions under pressure ( $<$ a few $\mathrm{kPa}$ ); (2) much higher energy can be used for molecular activation in comparison with that in thermal or photochemical reactions; (3) transfer of excessive energy in a single step might result in the formation of a considerable quantity of ionic species, which cannot be generated by any other method; and (4) excitation through the bombardment of energetic electrons would not be controlled by selection rules as in photochemistry and would; therefore, often lead to the occurrence of unusual reactive species of synthetic interest. In the following paragraphs, some organic reactions are discussed to exemplify the characteristics of chemical processes in nonequilibrium plasmas. For comprehensive reviews with respect to organic plasma chemistry, refer to the literature (Suhr 1983, 1989).

In plasmas, reactive species such as atomic hydrogen, oxygen and nitrogen are easily formed by the dissociation of molecular gases. While hydrogen and nitrogen atoms are mostly used in inorganic reactions, oxygen atoms are of special interest to organic reactions. When olefins or aromatic hydrocarbons were reacted in the plasmas of pure oxygen or air, the corresponding epoxides (Weisbeck and Hullstrung 1970) or phenols (Tezuka et al 1982) respectively could be produced in fairly good yields. Likewise, it is possible to generate radicals by the dissociation of suitable precursors. For example, $\mathrm{CF}_{3}$ radicals generated from hexafluoroethane were successfully reacted with metals or metal halides to synthesize a diversity of transition metal alkyl compounds (Lagow and Morrison 1980),

$$
\begin{aligned}
\mathrm{C}_{2} \mathrm{~F}_{6} & \rightarrow 2 \mathrm{CF}_{3}, \\
2 \mathrm{CF}_{3}+\mathrm{HgCl}_{2} & \rightarrow \mathrm{Hg}\left(\mathrm{CF}_{3}\right)_{2}+2 \mathrm{Cl}
\end{aligned}
$$

Another interesting reactive species is the $\mathrm{CN}$ radical which can be easily prepared from acetonitrile or cyanogen in plasmas. Benzene was converted to benzonitrile in $94 \%$ yield by using $(\mathrm{CN})_{2}$ plasmas (So and Miller 1981). Monosubstituted benzenes were also cyanated in respectable yields. In the reaction of ethylene or propylene with $\mathrm{CN}$ radicals, acrylonitrile was afforded in more than $70 \%$ yield (Henis and Miller 1982).

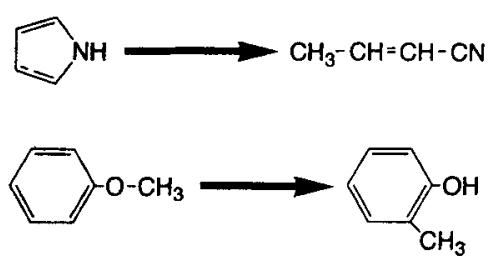




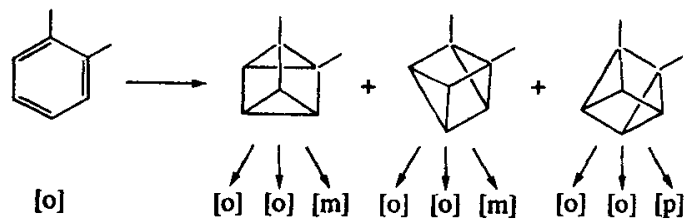

A number of unique isomerization reactions have been found in plasmas also. However, only a few examples of synthetic interest are illustrated here, that is, the isomerization of heterocyclic nitrogen compounds to unsaturated nitriles and the rearrangement of alkyl aryl ether to alkylphenols.

The transposition of the substituents on aromatic rings is also interesting, the intermediate formation of the prismane-type isomers based on the ratio of isomeric products from dicyanobenzenes was postulated (Yajima et al 1987).

One set of organic plasma reactions studied most extensively might be those of elimination, where small molecules, e.g., $\mathrm{H}_{2}, \mathrm{~N}_{2}, \mathrm{H}_{2} \mathrm{O}, \mathrm{CO}, \mathrm{CO}_{2}$ etc., were removed from

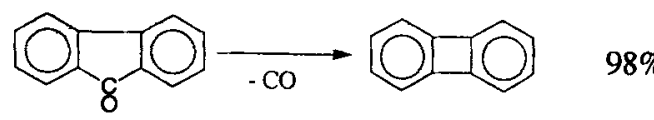<smiles>CC1C2CCC(C)(C1C)C2(C)C</smiles>

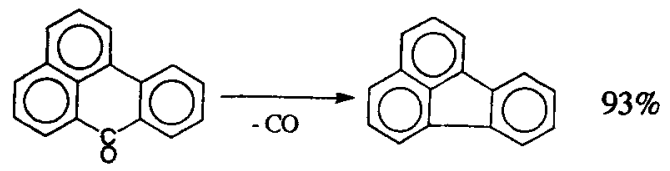

larger ones without drastic structural changes. Ring contractions, the generation of multiple bonds, ring formations and condensation reactions are especially attractive for preparative work. Some examples are shown above. In each case, the decarbonylation could be achieved in good yield (Suhr and Szabo 1975; Kruppa and Suhr 1980).

The desulfurization of benzothiophene or benzothiazole is also intriguing. In the latter case, the desulfurization resulted in the formation of benzonitrile. On account of the products from methylbenzothiazoles, the isonitriles, rather than the four-membered rings, were assumed to be the intermediates (Tezuka, unpublished results).

\section{Final remarks}

A number of organic plasma reactions of synthetic significance can be satisfactorily carried out on a laboratory scale. However, there have been only a few cases where the applications to industries were successfully executed. One of the causes is the low 


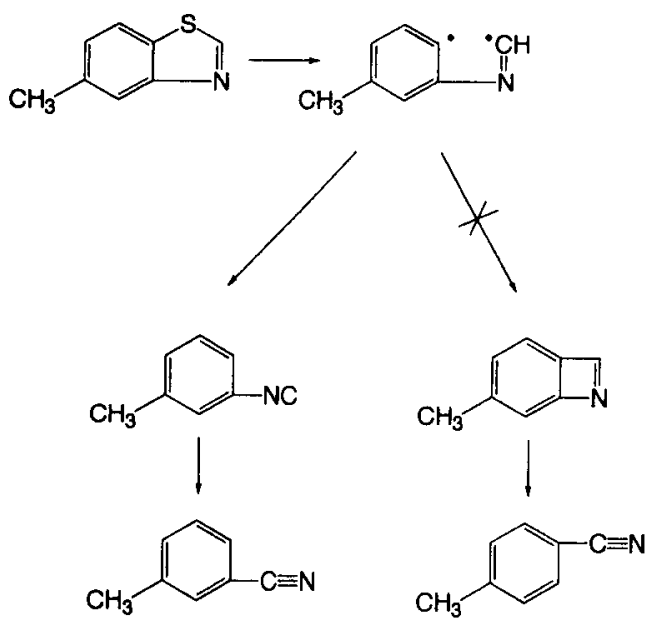

throughput, due to the intrinsic feature of most of the nonequilibrium plasmas being under low pressure. The scale-up of reactors might be crucial to resolve this problem. Another subject is the improvement of product selectivities. Generally, reactions in gaseous plasmas are not very selective, resulting from (1) similarity of bond strengths and ionization potentials in most organic compounds, (2) formation of "hot" products in the course of plasma reactions, and (3) overconversion of the desired products in plasmas. A promising approach to overcome these difficulties is to construct reaction systems involving plasma-liquid interactions, where the reactive species generated in a gaseous plasma can diffuse or migrate into the liquid in contact with the plasma and undergo quenching or reactions with the solvent and/or solutes.

Plasma chemistry is a truly interdisciplinary field. The role of chemists is growing in the elucidation of reaction mechanisms, in analysis, in development of new synthetic processes, and in providing novel starting materials for etching and coating processes.

\section{References}

Henis N B H and Miller L L 1982 J. Am. Chem. Soc. 1042526

Kruppa G and Suhr H 1980 Ann. Chem. 677

Lagow R J and Morrison J A 1980 Adv. Inorg. Chem. Radiochem. 23177

So Y H and Miller L L $1981 \mathrm{~J}$. Am. Chem. Soc. 1034204

Suhr H 1983 Plasma Chem. Plasma Process. 31

Suhr H 1989 Plasma Chem. Plasma Process. 9 7S

Suhr H and Szabo A 1975 Ann. Chem. 351

Tezuka M, Yajima T and Tsuchiya A 1982 Chem. Lett.. 1437

Weisbeck R and Hullstrung D 1970 Chem. Ing. Tech. 421303

Yajima T, Tsuchiya A and Tezuka M 1987 J. Chem. Soc., Chem. Commun. 1390 